\title{
The psychiatric manifestations of mitochondrial disorders: a case of catatonic presentation
}

\author{
C. Sereijo $^{1}$, R. Saraiva ${ }^{1}$, M.J. Gonçalves ${ }^{1}$, L. Linhares ${ }^{1}$, E. Fernandes ${ }^{1}$, L. Castanheira ${ }^{1}$, D. Costa Gomes ${ }^{2}$,R. Barandas ${ }^{1}$. \\ ${ }^{1}$ Centro Hospitalar Lisboa Norte - EPE, Psychiatry and Mental Health, Lisbon, Portugal. \\ ${ }^{2}$ Centro Hospitalar Lisboa Norte - EPE, Internal Medicine, Lisbon, Portugal.
}

\begin{abstract}
Objectives:
1. To report a case of mitochondrial cytopathy in a adult patient with catatonic presentation.

2. To make a brief literature review on mitochondrial cytopathy and catatonia treatment.
\end{abstract}

\section{Background:}

- Mitochondrial cytopathies represent a heterogeneous group of disorders that affect energy-dependent organs such as the brain. ${ }^{1.2 .3}$

- Patients with mitochondrial disorders can present with primary psychiatric symptomatology, including mood disorder, cognitive impairment, psychosis and anxiety, particularly those with medical comorbidities. ${ }^{1.2 .3}$

\section{Materials and Methods:}

Case description with clinical information. Non-systematic review of the literature (MEDLINE).

\section{Case presentation:}

- Identification: forty-six year old woman, with the diagnosis of mithocondrial citopathy with a MFN2 (c.1724G>A) heterozygotic mutation that is currently on investigation.

- Past medical history: of multiple problems affecting several energy-dependent organs such as the brain (cognitive impairment, refractory seizures), heart (hypertrophic cardiomyopathy), and muscle (fatigue and atrophy), and the presence of other key features of mitochondrial disorders, such as short stature, diabetes mellitus and sensorineural hearing loss.

- Psychiatric history: cognitive impairment, disruptive behavior.

- Previous medication: topiramate $200 \mathrm{mg}$ 2id, perampanel $4 \mathrm{mg}$, azatioprine $50 \mathrm{mg}, 25 \mathrm{mg}$ pantoprazol $20 \mathrm{mg}$ e insuline.

- Current history: The patient was stable until two months before her psychiatric hospitalization when she started with verbal agressivity towards her parents, anorexia and lack of higiene. She had a recent initiation of perampanel for epilepsy. She was hospitalized in October/2018 at the Internal Medicine department for anorexia (refuse to eat) and psychomotor agitation. After medical stabilization she was transferred to the psychiatry unit.

- Mental status examination at the admission: "low cooperation, oriented in all spheres and presented passive negativism. Establishes eye contact and has higher speed of blinking. Psychomotor retardation. Laconic, slow and delayed speech, not spontaneous. Sub depressive mood.”

- Complementary diagnostic exams: CEMRI: "small hyperintense in the white substance that appear to be of microvascular nature".

- Treatment and evolution: she initiated treatment with mirtazapine and quetiapine and had a new episode of seizures and had to stop this treatment. We started treatment with lorazepam with few results and then we switched to amantadine up to $200 \mathrm{mg} /$ day with gradual improvement of her clinical state, however, not returning to the previous cognitive and functional state. She was observed by the movement disorder neurology team that decided to initiate levodopa $100 \mathrm{mg}+$ carbidopa $25 \mathrm{mg}$ because of her bradykinesia and other parkinsonian like motor symptoms with some improvement.

\section{Results and Conclusions:}

- Catatonia is an episodic syndrome characterized by specific affective, behavioral, and motor symptoms and thought to result from regional abnormalities in neurotransmitter function. It can be caused by a number of psychogenic, infectious, iatrogenic, and neurological conditions. 4,5

- Mithocondrial disorders have only recently been described, with the first pathogenic mutation identified in $1988 .{ }^{3}$

- The psychiatric presentation of mitochondrial disorders has been under recognized in the fields of mitochondrial medicine and psychiatry. ${ }^{1.2 .3}$

- This diagnosis has important implications since many psychotropic medication might impair mitochondrial function or have side effects that may worsen medical conditions associated with mitochondrial disorders. 1,3 